\title{
Variation in Mass of Entities in Condensed Media
}

\author{
Volodymyr Krasnoholovets \\ Indra Scientific SA, Square du Solbosch 26 \\ B-1050 Brussels, Belgium \\ Tel: 32-2-640-27-51 E-mail: v_kras@yahoo.com
}

\begin{abstract}
The present paper further develops some previous results associated with the variation in mass of a system starting from first sub microscopic principles and the constitution of real space. The wave function $\psi$ determined in a phase space is treated as an image of the original field of inertia determined in the real space, which is generated by the moving particle. Carriers of this field, inertons, are responsible for the exchange of mass between the substance's entities (atoms or molecules that move or vibrate in the substance). The overlapping of inerton clouds results in the emission and re-absorption of inertons by vibrating entities. Thus the mass of atoms in a substance is not a stationary parameter, but dynamic. Methods of submicroscopic mechanics allow a detailed study of a mass dynamics in the substance in question. Moreover, the inerton field can be excited in a substance and can affect other substances inducing new effects, such as 'freezing' and clusterization of entities, which can give rise to new chemicals. Besides, those are inertons that synchronize the coherent motion of ultracool atoms bringing them to the cluster state, which is identified with the phenomenon of Bose-Einstein condensation.
\end{abstract}

Keywords: Variation in mass, Inerton field effects, 'Frozen' molecules, Clusterization, Cool atoms, Bose-Einstein condensation

\section{Introduction}

Bounias and Krasnoholovets $(2003,2004)$ proposed a detailed mathematical theory of the constitution of the real physical world. In line with this theory, real space is constrained to be a mathematical lattice of closely packed topological balls with approximately the Planck size, $\left(\hbar G / c^{3}\right)^{1 / 2} \sim 10^{-35} \mathrm{~m}$. It was proven that such a lattice is a fractal lattice and that it also manifests tessellation properties. It has been called the tessel-lattice. In the tessel-lattice, a ball is allowed both volumetric and surface fractalities, which are associated respectively with such fundamental physical concepts as mass (Bounias \& Krasnoholovets, 2003b) and charge or electromagnetism in general (Krasnoholovets, 2003). This view is consistent with ordinary studies on fractal geometry. For instance, Feder (1998) writes that a curve can be measured by means of the number of balls that cover it; this in fact is a first crude step to the concept presented by Bounias and Krasnoholovets $(2003,2004)$. Thus, the tessel-lattice allows one to completely replace such uncertain notions as ether, physical vacuum, space-time, urfield, cosmic plenum, ultimate referent, ultra-referential reality, energy substrate, etc. and hence to make clear the science behind the so-called first principles sustained by orthodox quantum theories.

A stable local deformation of the tessel-lattice, i.e. its fractal deformed cell, is treated as a canonical particle. Subatomic mechanics, or submicroscopic mechanics of the motion of a particle in the tessel-lattice developed in a series of works (see the review paper by Krasnoholovets, 2003a) has a direct comparison with conventional quantum mechanics, which, as is well known, operates in an abstract phase space. Submicroscopic mechanics is developed in real space and represents an inner sub structure of the $\psi$-wave function giving expressions that interlink the particle's de Broglie wavelength $\lambda$ with the range of space $\Lambda$ covered by the spatial excitations that accompany the moving particle; in particular, the amplitude of inerton cloud is tied with the de Broglie wavelength by means of relationship

$$
\Lambda=\lambda c / v
$$

where $c$ is the velocity of light (for a charged particle) and $v$ is the velocity of the particle. These excitations have been called inertons due to the fact that they represent carriers of the force of inertia, i.e. motion as such (from one understanding), or a resistance that emerges on the side of space when a material object is moving through the space, which in our theory is treated as the tessel-lattice (from the other interpretation).

The inerton cloud of an entity (a particle, atom or molecule) spreads to the length $\lambda / 2$ along the entity's path 
and occupies the area $\pi \Lambda^{2}$ in transversal directions.

The reality of inertons has been proved experimentally in many different physical systems: changes in the fine morphological structure of a metal (Krasnoholovets and Byckov, 2000); the anomalous photoelectric effect (Krasnoholovets, 2001a); cooperation of hydrogen atoms (Krasnoholovets, 2001b); freezing of amplitudes of fluctuating molecules in aqueous solutions (Krasnoholovets, Skliarenko \& Strokach, 2006; Andreev, Dovbeshko \& Krasnoholovets, 2007); binding of electrons into electron droplets (Krasnoholovets, Kukhtarev, Kukhtareva, 2006) where, based on rigorous experimental results it was shown that the formation of stable electron clusters containing around $10^{10}$ electrons occurred due to the absorption of inerton radiation by photoelectrons where the inerton radiation originated from the crystal surface illuminated by a laser beam. The measurement of inerton radiation (a mass flow falling on the unit surface per second) from different sources in a laboratory, the Earth and the sky has been carried out in paper (Didkovsky \& Krasnoholovets).

The present paper develops some of the previous results associated with a variation in mass of the system in question. In particular, the necessity of linking of the mass-energy relation $\pm \Delta m c^{2}$ to the laws of thermodynamics was shown in our paper (Krasnoholovets \& Tane, 2006); our experimental work (Andreev, Dovbeshko \& Krasnoholovets, 2007) concluded that the defect of mass $\Delta m$ appeared in the chemical physical system studied. Hence $\Delta m$ becomes an inner property not only of atomic nuclei, but of any physical and physical chemical systems. By changing the mass of particles, inertons, as carriers of local deformations of space, make an entry into the field mechanics. Indeed, the introduction of inertons immediately destroys the principle of uncertainty

$$
[\Delta x, \Delta p] \geq \hbar
$$

because instead of one undetermined "wave-particle" we have two subsystems: the particulate cell (the particle kernel) and the inerton cloud that accompanies it, although the uncertainty relation above takes into account only the particle (i.e. the particulate cell). The 'particle-inerton cloud' system determined in the real space cannot be presented as a package of superimposed monochromatic abstract waves. But it is this approximation that leads to the inequality of wave numbers $\Delta k_{i}$ and the appropriate variations in coordinates $\Delta r_{i}: \Delta k_{x} \Delta x \geq \eta$, $\Delta k_{y} \Delta y \geq \eta$, and $\Delta k_{z} \Delta z \geq \eta$ where $\eta \approx 1$ is a parameter; then substituting $\Delta k_{i}$ for $p_{i} / h$ we have $\Delta p_{x} \Delta x \geq h, \Delta p_{y} \Delta y \geq h$ and $\Delta p_{z} \Delta z \geq h$ (read de Broglie, 1982).

Hans G. Dehmelt who won the Nobel Prize 1989 for the development of the ion trap technique experiments proved together with co-workers (Dehmelt \& Ekstrom, 1973; Wineland, Ekstrom \& Dehmelt, 1973; van Dyck, Ekstrom, \& Dehmelt, 1976; van Dyck, Schwinberg \& Dehmelt, 1986) that both the position and momentum of an electron could be measured simultaneously; he and his collaborators kept a practically motionless electron in an electromagnetic confinement system for months, which allowed them to measure simultaneously - with accuracy $10^{-11}$ to $10^{-16}$ - the position and momentum, and also other parameters.

In submicroscopic mechanics developed by the author the momentum $p$ of a particle is decomposed to the mass $m$ and the velocity $v$ and each of these parameters is characterized by its own behavior in the line of a particle's path. The whole particle path is subdivided by the particle's de Broglie wavelength $\lambda$ : along the section $\lambda$ the particle velocity changes from $v \rightarrow 0$ and is reinstated to $v$ (for details see Krasnoholovets, 2002a); owing to the emission and re-absorption of inertons by the particle, its mass also varies, $m \rightarrow 0 \rightarrow m \rightarrow 0 \ldots$ (Krasnoholovets, 2002b), because inertons transfer fragments to the total mass of the particle: due to the motion a local deformation of space (which is a volumetric fractal deformation of a cell, i.e. mass $\mathrm{m}$ ) periodically changes to a local tension of space (which is a displacement of the volumetric fractal deformation from its equilibrium state, $\Xi$ ). Furthermore, it was revealed that periodical transformations of physical parameters of quantum systems from the original state to a tension state are universal and such transformations occur just in the section $\lambda$ of a particle path. For instance, a periodical pulsation of the shape of the particulate cell (the particle kernel) between the beanlike and spherical form is associated with the notion of particle spin (Krasnoholovets, 2002a); the periodical conversion of the surface state of the particle kernel from the chestnut shape to the inclined needles shape corresponds to the electric charge and the monopole, respectively, which is described by the Maxwell equations (Krasnoholovets, 2003). In the case of a free photon (Krasnoholovets, 2003), which moves hopping from cell to cell, the section $\lambda_{\text {photon }}$ equals to its wavelength, the photon's electric polarization (which stands for fractal needles normal to the surface of a coming cell) 
periodically transforms to the magnetic polarization (in this state fractal needles are inclined, such that they are tangential to the surface).

In this paper a dispersion law $\omega(k)$ is derived describing the oscillation of the value of mass of atoms/molecules in a lattice of entities. Then it is shown how this oscillating mass field assists the lattice in the generation of clusters. Finally we study the dependence of the number of entities in a cluster on the external inerton field (i.e. a mass field) and show how the outside inerton field becomes a control field of interactions in the system in question.

\section{Behavior of mass}

In compliance with submicroscopic mechanics (Krasnoholovets, 2002a), the core of a moving particle is accompanied by a cloud of the particle's inertons ejected from the particle on it's collisions with cells of the space tessel-lattice. In condensed media, atoms/molecules vibrate near their equilibrium positions. The crystal lattice is a good model to reveal major regularities in a dynamics of condensed matter and that is why this model is used below.

In the crystal lattice the behavior of nodes is defined by the Lagrangian

$$
L_{\mathrm{vibr}}=\frac{1}{2} \sum_{\mathbf{n}}\left(m_{\mathbf{n}} \delta \dot{r}_{\mathbf{n}}^{2}-\gamma \delta r_{\mathbf{n}}^{2}\right)
$$

where $m_{\mathbf{n}}$ is the mass of the $\mathbf{n}$ th node, $\delta r_{\mathbf{n}}$ is the deviation of this node from its equilibrium position and $\gamma$ is the force constant. It is well known that based on the Lagrangian (2) the Euler-Lagrange equations can be constructed, which completely determine the dynamics of the nodes.

Since massive nodes vibrate near their equilibrium positions, i.e. are in motion, they emit and re-absorb clouds of inertons. Therefore inertons periodically remove a part of the mass from vibrating nodes and bring it back. Such behavior can be described in terms of the Lagrangian below (for simplicity of consideration we consider the one-dimensional lattice), which is similar in form to the expression (2) but is different in dimension, as its variables are masses

$$
L_{\text {mass }}=\sum_{\mathbf{n}}\left\{\frac{1}{2} \dot{m}_{\mathbf{n}}^{2}-\frac{\pi}{2 T}\left(\dot{m}_{\mathbf{n}} \mu_{\mathbf{n}}+\dot{m}_{\mathbf{n}+\mathbf{g}} \mu_{\mathbf{n}}\right)+\frac{1}{2} \dot{\mu}_{\mathbf{n}}^{2}\right\}
$$

where $m_{\mathbf{n}}$ and $\mu_{\mathbf{n}}$ are variations of mass of $\mathbf{n t h}$ node and its cloud of inertons, respectively, which occur due to the overlapping of inerton clouds of neighbor nodes; $\mathbf{g}$ is the lattice vector; $T$ is the period of collision of the mass located in the $\mathbf{n}$ th node with its inerton cloud. The dot over mass means the derivative in respect to the time $t$ treated as a natural parameter.

Instead of variables $m_{\mathbf{n}}$ and $\mu_{\mathbf{n}}$ we may pass on to collective variables $\Phi_{\mathbf{k}}$ and $\phi_{\mathbf{k}}$ by rules

$$
\begin{aligned}
& m_{\mathbf{n}}=\frac{1}{\sqrt{N}} \sum_{\mathbf{k}} \Phi_{\mathbf{k}} e^{i \mathbf{k g}} \\
& \mu_{\mathbf{n}}=\frac{1}{\sqrt{N}} \sum_{\mathbf{k}} \phi_{\mathbf{k}} e^{i \mathbf{k g}}
\end{aligned}
$$

Substituting expressions (4) and (5) into the Lagrangian (3), we obtain

$$
L_{\text {mass }}=\frac{1}{N} \sum_{\mathbf{n}}\left\{\frac{1}{2} \dot{\Phi}_{\mathbf{k}} \dot{\Phi}_{-\mathbf{k}}-\frac{\pi}{2 T}\left(\dot{\Phi}_{\mathbf{k}} \phi_{-\mathbf{k}}+\dot{\Phi}_{\mathbf{k}} \phi_{-\mathbf{k}} e^{i \mathbf{k} \mathbf{g}}\right)+\frac{1}{2} \dot{\phi}_{\mathbf{k}} \dot{\phi}_{-\mathbf{k}}\right\}
$$

In the Lagrangian (5) each summand must be real, in particular, the term $\dot{\Phi}_{\mathbf{k}} \phi_{-\mathbf{k}}$. Then in the term $\dot{\Phi}_{\mathbf{k}} \phi_{-\mathbf{k}} e^{i \mathbf{k g}}$ the exponent $e^{i \mathbf{k g}}$ must also be real. This means that the Lagrangian (6) is real and reduces to

$$
L_{\text {mass }}=\frac{1}{N} \sum_{\mathbf{k}}\left\{\frac{1}{2} \dot{\Phi}_{\mathbf{k}} \dot{\Phi}_{-\mathbf{k}}-\frac{\pi}{2 T}(1+\cos \mathbf{k g}) \dot{\Phi}_{\mathbf{k}} \phi_{-\mathbf{k}}+\frac{1}{2} \dot{\phi}_{\mathbf{k}} \dot{\phi}_{-\mathbf{k}}\right\}
$$

The Euler-Lagrange equations

$$
\frac{d}{d t} \frac{\partial L_{\text {mass }}}{\partial \dot{q}}-\frac{\partial L_{\text {mass }}}{\partial q}=0
$$


where the variable $q$ stands for $\Phi_{\mathbf{k}}$ and $\phi_{\mathbf{k}}$, become

$$
\begin{gathered}
\ddot{\Phi}_{\mathbf{k}}-\omega(k) \dot{\phi}_{-\mathbf{k}}=0, \\
\ddot{\phi}_{\mathbf{k}}+\omega(k) \dot{\Phi}_{\mathbf{k}}=0
\end{gathered}
$$

where we designate

$$
\omega(k)=\frac{\pi}{2 T}(1+\cos (\mathbf{k g}))
$$

Periodical solutions to equations (8) and (9), which satisfy the physical characteristics of the system of varying masses, can be chosen as follows

$$
\begin{aligned}
& \Phi_{\mathbf{k}}=\Phi_{0}+\Phi_{1} \cos (\omega k(t)), \\
& \phi_{\mathbf{k}}=-\Phi_{1} \cos (\omega(\mathbf{k}) t)
\end{aligned}
$$

where, as follows from expressions (4) and (5), parameters $\Phi_{0}$ and $\Phi_{1}$ are proportional to the rest mass of the system's particles and the mass of their inerton clouds, respectively, and inversely proportional to the square root of the total number of particles $N^{-1 / 2}$.

The mentioned arguments point out that the variables $\Phi_{\mathbf{k}}$ and $\phi_{\mathbf{k}}$ represent collective massive excitations in the lattice: $\Phi_{\mathbf{k}}$ describes collective mass excitations of the nodes of the lattice $\phi_{\mathbf{k}}$ characterizes the mass field of inertons that like dust fill the entire space between the nodes in the lattice. The spectrum of these collective mass excitations, is the dispersion law (10) that in the long-wave approximation becomes

$$
\omega(k) \cong \frac{\pi}{T} \cdot\left(1+\frac{1}{2}(\mathbf{k g})^{2}\right) .
$$

It should be emphasized that these mass excitations are completely independent from the phonons of the lattice, be cause phonons are associated with collective changes of positions of nodes (atoms). Mass excitations described by the variable $\Phi_{\mathbf{k}}$ represent the collective mass state of nodes at the moment $t$ and the variable $\phi_{\mathbf{k}}$ depicts the collective state of the total inerton cloud of the lattice.

The amplitude $\delta m$ of oscillations of the nth node's mass can crudely be estimated as a ratio of the dispersion of the $\mathbf{n}$ th node's inerton cloud at the maximal distant object $(r=\Lambda)$ and the nearest node $(r=g)$

$$
\delta m \approx m_{\mathbf{n}} \frac{g}{\Lambda} \sim 10^{-4} m_{\mathbf{n}}
$$

where $\Lambda$ is the amplitude of inerton cloud of the $\mathbf{n}$ th node. In accordance with correlation (1), the mentioned amplitude is related to the node's de Broglie wavelength, $\lambda_{\mathbf{n}} \equiv \delta r_{\mathrm{n}}$, the node's velocity (sound velocity) $v$ and the inerton velocity in the lattice can be equal to the velocity of light, $c$; then

$$
\Lambda_{\mathrm{n}}=\delta r_{\mathrm{n}} \frac{c}{v} \geq \delta r_{\mathrm{n}} \cdot 10^{5} \sim 10^{4} \mathrm{~g}
$$

where we put approximately that the amplitude of oscillations $\delta r$ of an node in the lattice is about 0.1 of the lattice constant $g$. The evaluation (15) results in the estimate (14).

\section{New kind of interaction in condensed media}

\section{1 'Frozen'molecules}

In paper (Krasnoholovets, 2004) the cluster formation in water was studied in the framework of a statistical mechanical approach in which the pair potentials of attraction and repulsion played the governing role. The infrared spectrum of water shows strong activity in the range of the hydrogen bond around $200 \mathrm{~cm}^{-1}$, which means that water molecules are characterized by a strong ionic polarizability and then the study of the statistical behavior of a water systems might be treated in the framework of a typical ionic lattice. Hence the pair potential of interacting water molecules may consist of an ionic crystal potential (which includes Madelung's constant $\alpha$ that falls within the range from unity to two, see e.g. Kittel, 1978), the potential of dipole-dipole interaction and a fluctuation quantum potential: 


$$
V_{\text {att }}(\rho)=\frac{\alpha e^{2}}{4 \pi \varepsilon_{0} \varepsilon(g, v) g \rho}+\frac{\sqrt{2 / 3}}{4 \pi \varepsilon_{0} \varepsilon(g, v)} \frac{d^{2}}{g^{3} \rho^{3}}-\frac{1}{2} \gamma(\delta r)^{2} \rho^{2}
$$

(note the correct sign of $V_{\text {att }}$ is opposite to that in expression (16) and also below in (23) and (28), which is included in processing of the action $S$ (Krasnoholovets \& Lev, 2003)). Here the second term in the right hand side of expression (16) is written for the average dipole-dipole interaction when all reciprocal orientations of dipoles are equi-probable (Agranovich \& Galanin, 1978).

The last term in the right hand side of expression (16) is the direct consequence of the availability of inerton clouds around moving/vibrating material entities, as discussed above, because, as it follows from expressions (14) and (15), the cloud of inertons spreads about six orders of the lattice constant's size. Therefore, the same entities covered by the same inerton clouds must demonstrate an elastic behavior. This last term was presented also in paper by Krasnoholovets (2004) and it was crucial for the derivation of the collective states of molecules.

In expression (6) $d$ is the dipole momentum of a node in the water network/lattice; $\varepsilon(g, v)$ is the permittivity as a function of frequency $v$ and the lattice constant $g ; \gamma$ is the force constant. In the right hand side of the potential $V_{\text {att }}(16)$, in the first term the distance $r$ from the node to a distant point is written as $r=g \rho$ and in the third term the amplitude of oscillations is depicted as $\delta r \rho$ where $\rho$ is the dimensionless distance.

The potential of repulsion can be chosen in any form, because it does not play a principal role in a solution describing a collective state (due to the fast-falling character of the potential). Let us choose it in Lennard-Jones' form

$$
V_{\text {rep }}(\rho)=V_{0} /(g \rho / g)^{12}=V_{0} / \rho^{12}
$$

Then in the case of an ensemble of $N_{\text {total }}$ particles (nodes of a lattice), we can use the preliminary results obtained in paper (V. Krasnoholovets, 2004). The action $S$ for the ensemble of $N_{\text {total }}$ interacting particles, which obey the Boltzmann statistics and tend to clusterization, becomes (Krasnoholovets, 2004)

$$
S=K \cdot[2 N \cdot(a-b)+N \ln \xi]
$$

where $N_{\text {total }}=K \cdot N$ and $N$ is the number of particles that try to form a cluster and $K$ is the number of possible clusters in the system of $N_{\text {total }}$ interacting particles; $\xi$ is the fugacity. The functions $a$ and $b$ look as follows ( $\Theta$ stands for the absolute temperature)

$$
\begin{aligned}
& a(N)=\left(3 / k_{\mathrm{B}} \Theta\right) \int_{1}^{N^{1 / 3}} V_{\text {rep }}(\rho) \rho^{2} d \rho \\
& b(N)=\left(3 / k_{\mathrm{B}} \Theta\right) \int_{1}^{N} V_{\text {att }}(\rho) \rho^{2} d \rho
\end{aligned}
$$

Substituting potentials (16) and (17) into expressions (19) and (20), respectively, and then inserting the functions $a$ and $b$ in explicit forms into Eq. (18) we obtain the following expression for the action $S$ of a cluster

$$
\begin{aligned}
S / K= & \frac{2}{3} \frac{V_{0}}{k_{\mathrm{B}} \Theta} N-\frac{3 \alpha e^{2}}{4 \pi \varepsilon_{0} \varepsilon(g, v) g} N^{5 / 3} \\
& -\frac{\sqrt{2 / 3} d^{2}}{2 \pi \varepsilon_{0} \varepsilon(g, v) g^{3} k_{\mathrm{B}} \Theta} N \ln N+\frac{3}{5} \frac{\gamma \delta r^{2}}{k_{\mathrm{B}} \Theta} N^{8 / 3}+N \ln \xi .
\end{aligned}
$$

The equation for the number of particles combined in a cluster comes from the equation $\partial S / \partial N=0$ if the inequality $\partial^{2} S / \partial N^{2} \mid \quad N=N_{\text {in cluster }}>0$ holds. Explicitly, 


$$
\begin{aligned}
N^{5 / 3} & -\frac{25}{8} \frac{\alpha e^{2}}{4 \pi \varepsilon_{0} \varepsilon(g, v) g \gamma \delta r^{2}} N^{2 / 3} \\
& -\frac{5 \sqrt{2 / 3}}{4} \frac{d^{2}}{4 \pi \varepsilon_{0} \varepsilon(g, v) g^{3} \gamma \delta r^{2}}(\ln N+1)+\frac{5 V_{0}}{12 \gamma \delta r^{2}}+\frac{5 k_{\mathrm{B}} \Theta}{8 \gamma \delta r^{2}} \ln \xi=0 .
\end{aligned}
$$

This equation can be solved numerically, for instance for the case of water. For water, the numerical values of the corresponding parameters are: $d=1.84$ Debye $=6.17 \times 10^{-30} \mathrm{C} \cdot \mathrm{m}$; Madelung's constant $\alpha=1.7$; permittivity $\varepsilon(g, v)=5$ (experimental examinations by Strobbe \& Peschel (1997) show that the dielectric constant $\varepsilon$ changes from 5 to 10 when the thickness of water layer varies from 1 to $12 \mathrm{~nm}$ ); the lattice constant, which also determines the effective size of a molecule in the lattice, $g \cong 2.8 \times 10^{-10} \mathrm{~m} ; \delta r$, as the amplitude of vibration of a molecule, can also be considered as an amplitude of fluctuation of the size of a molecule in the lattice and, as the rule $\delta r \sim 10^{-11} \mathrm{~m}$.

In Eq. (22) the fugacity $\xi=\exp \left[-\mu /\left(k_{\mathrm{B}} \Theta\right)\right]$; the chemical potential $\mu$ in the first approximation can be chosen as is the case for the gas phase $\mu=3 k_{\mathrm{B}} \Theta \ln \left(\langle\lambda\rangle n^{1 / 3}\right)$ where $n=3 \times 10^{28} \mathrm{~m}^{-3}$ is the concentration of water molecules and the de Broglie's thermal wavelength $\langle\lambda\rangle=h /\left(3 m k_{\mathrm{B}} \Theta\right)^{1 / 2}$. The mass of a water molecule $m=2.99 \times 10^{-26} \mathrm{~kg}$ and hence putting $\Theta=300 \mathrm{~K}$ we get $\langle\lambda\rangle \cong 3.4 \times 10^{-11} \mathrm{~m}$, and thus $\langle\lambda\rangle n^{-1} \cong 0.105648$. The initial potential of repulsion $V_{0}$ can be chosen as an average value of the energy of a hydrogen bond in water and at room temperature $V_{0} \approx 10 k_{\mathrm{B}} \Theta_{300} \cong 4.25 \times 10^{-20} \mathrm{~J}$ where $\Theta_{300 .}=300 \mathrm{~K}$. The force constant $\gamma$ of the water network is a fitting parameter, the value of which is anticipated in the order of $1 \mathrm{~N} / \mathrm{m}$ (in solids $\gamma$ varies approximately from $1 \mathrm{~N} / \mathrm{m}$ to tens of $\mathrm{N} / \mathrm{m}$ ); below we put $\gamma=1 \mathrm{~N} / \mathrm{m}$.

Fig. 1 depicts the solution to Eq. (22) as a function of amplitude $\delta r$. We can see the lesser the value of amplitude $\delta r$, the large the number of particles involved in a cluster. The other solution for $N$ falls within the interval from 0 and 1, which signifies that the considering system of $N_{\text {total }}$ water molecules represents a kind of a mixture of two phases: clusters and individual molecules.

Let us examine a model system of $N_{\text {total }}$ molecules interacting through the Lennard-Jones pair potential. In this case taking into account the inerton interaction $\gamma \delta r^{2} / 2$ between molecules the attraction and repulsion parts of the pair potential respectively are

$$
V_{\mathrm{att}}=\frac{V_{0}}{\rho^{6}}-\frac{1}{2} \gamma(\delta r)^{2} \rho^{2}
$$

and expression (17) for $V_{\text {rep }}$. For these potentials the action (18) has the form

$$
S / K=-\frac{4}{3} \frac{V_{0}}{k_{\mathrm{B}} \Theta} N+\frac{3}{5} \frac{\gamma \delta r^{2}}{k_{\mathrm{B}} \Theta} N^{8 / 3}+N \ln \xi
$$

and the equation for the number of molecules in a cluster $\partial S / \partial N=0$ results in the solution (if we neglect the contribution on the side of $\ln \xi$ )

$$
N \approx\left(\frac{5}{6} \frac{V_{0}}{\gamma \delta r^{2}}\right)^{3 / 5} .
$$

The dependence of $N$ as a function of $\delta r$ is shown in Fig. 2.

The appearance of fractal clusters, as it follows from the fractional degree of the major term in Eq. (22) and also in expression (25), significantly changes the behavior of the system. In particular, we can see the lesser $\delta r$, the larger $N$. However, this is possible only when the amplitude $\delta r$ of oscillations of particles near their equilibrium positions begins to drop, i.e. becomes less than approximately $10^{-11} \mathrm{~m}$.

The question arises under what conditions can $\delta r$ drop if the temperature does not decrease? Such conditions can be realized by means of an incident inerton radiation. Indeed, as has been shown in section 2 , since the mass of entities oscillates in a substance (i.e. emits and re-absorbs fragments of mass), it can also absorb incident inertons that carry mass.

It was shown in papers (Krasnoholovets, 2002b; Krasnoholovets, Skliarenko \& Strokach, 2006; Andreev, 
Dovbeshko \& Krasnoholovets, 2007; Krasnoholovets, Kukhtarev \& Kukhtareva, 2006) that the inerton field revealed strong interactions with substances. In particular, experimental studies (Krasnoholovets, Skliarenko \& Strokach, 2006a) disclosed that the influence of inerton field on water was similar to the induction of an additional value of the dipole moment in a water molecule. However, this is only the first phenomenological supposition. A submicroscopic analysis has indicated that the incident inerton field induced weight modifications of water molecules: Aqueous solutions strongly change their behavior under the application of the inerton field, compare Figs. 3a and 3b and see Fig. 4 (Krasnoholovets, Skliarenko \& Strokach, 2006a). The same was observed in the case of electron droplets: Electrons underwent a huge weight modification, such that their mass millions of times exceeded the rest mass of free electron. Consequently, physical entities are able to absorb inertons, which results in the entity's increased mass. Hence, the amplitude of the entity's vibrations decreases:

$$
m \rightarrow m+\Delta m, \quad \delta r=h /(m v) \rightarrow \delta r^{\prime}=h /[(m+\Delta m) v], \quad \text { that is, } \quad \delta r^{\prime}<\delta r .
$$

The comparison of Fig. 3a and Fig. 3b shows that during the first 15 minutes when the alcohol has not yet significantly evaporated from the aqueous solution, water molecules become frozen - the curve in Fig. $3 \mathrm{~b}$ shows an anomaly small capacity, which is the results of 'frozen' molecules in the aqueous solution (Krasnoholovets, Skliarenko \& Strokach, 2006a). Fig. 4 shows the same: the lower curve means freezing of molecules in the solution caused by the absorption of inertons.

So, an incident inerton field is absorbed by the atoms/molecules of the substance studied, which decreases the amplitude $\delta r$ of their vibrations in line with relations (26). Even a small reduction of $\delta r$ is capable of significantly changing the rearrangement of entities, which gives rise to a redistribution of entities into clusters increasing the cluster size (see Fig. 2). In clusters weighted atoms/molecules are able to exchange mass, as has been described in section 2, and hence the frequency range and the intensity of the incident inerton field may impose the appropriate behavior of entities in a cluster. As follows from our experimental studies (Krasnoholovets, Skliarenko \& Strokach, 2006; Andreev, Dovbeshko \& Krasnoholovets, 2007; Krasnoholovets, Kukhtarev \& Kukhtareva, 2006; Krasnoholovets \& Tane, 2006), such new massive-cluster states are rather long-living; extending to at least hours.

It seems the phenomenon discussed is widely encountered in nature. For instance, when the atmospheric conditions become unstable, inertons may influence the gases of the air. In this case a new distribution of clusters in gases will be established inducing new dynamic properties of the air. Therefore, during unstable weather conditions, living entities come into contact with such perturbed air and the influence of inertons on the entities becomes stronger, which may explain why some people are predisposed/more sensitive to changes in weather conditions (the blood of sensitive people becomes more dense, or rather viscous, in the periods of so-called magnetic storms when a compass needle is dancing, which is observed at the appearance of black spots on the sun).

The absorption of inertons resulting in a new cluster formation may be considered as the main principle of homeopathy and its modern more powerful branch of medicine called information medicine (electro-acupuncture points having bad conductivity can be corrected through injection of the mass field, or the field of spatial deformations, i.e. the inerton field, in a special frequency range, which corresponds to oscillations of the organ in question).

Different frozen water crystallites obtained and investigated by Emoto's team (Emoto, 2005), which were formed under different environmental conditions (various pictures or sounds, such as music, prayer, unpleased words, etc.), could be associated only with the presence of an incident inerton radiation; this radiation should be generated in parallel with acoustic waves, as has been argued above. The appearance of Emoto's water clusters can be associated with the supplement elastic inerton interaction $\gamma(\delta r)^{2} / 2$ between water molecules (see last terms in expressions (16) and (23)). A variety of shapes of snowflakes we can obtain on the basis of expressions (18)-(20) if we assume an asymmetry of the inerton field, i.e. the term $\gamma(\delta r)^{2} / 2$ in the mentioned formulas has to be substituted for $\gamma_{x}(\delta x)^{2} / 2, \gamma_{y}(\delta y)^{2} / 2$ and $\gamma_{z}(\delta z)^{2} / 2$. However, this mathematical problem requires a separate study.

In the chemical industry, inerton fields are able to play the role of a field catalyst or, in other words, inerton fields can serve to control the speed of chemical reactions. Namely, in a reactive chamber inerton fields 'freeze' molecules of the reacting substances. If in the reactive chamber substances move rapidly, the motion will hasten reactions of breaking of long 'frozen' molecules into shorter molecules. This procedure does not require high 
temperature and high pressure or long term mechanical mixing (hours), which are typical for modern chemical technologies. Under the inerton radiation, the formation of a new substance takes place in a few seconds. One such application of inerton fields is in the manufacture of biodiesel from a medley of vegetable oil and methanol (with catalyst $\mathrm{KOH}$ ), which has been described in our patent application (Dekhtiaruk, Krasnoholovets \& Heighway, 2007).

It is interesting to note that other researchers seem to encounter inerton fields when dealing with practical applications, especially in the area of new energy technologies. For instance, Santilli (2001) wrote that their fuel synthesis gas called 'magnegas' did not show any activity in the infrared spectral range. Such a claim is precisely consistent with these studies: even a weak inerton signal generated by a Teslar ${ }^{\circledR}$ watch is able to significantly decrease the intensity of the reflectance signal of the substance (the lower curve in Fig. 4). Therefore one can anticipate that a strong inerton field is capable of holding down the spectral line of the substance studied to the wave number-axis in Fig. 4, which is in agreement with the case described by Santilli (2001), i.e. no response to an infrared excitation of their samples.

\subsection{Cool atoms, trapping states and the Bose-Einstein condensation}

The discovery of laser cooling, trapping states and the Bose-Einstein condensation in dilute gases of atoms started a number of new areas of research (Chu, 1998; Cohen-Tannoudji, 1998; Phillips, 1998; Cornell \& Wieman, 2002; Ketterle, 2002). Generally the discovered phenomena represent collective effects in a quantum gas. By now seem the cooling mechanism and the Bose-Einstein condensation have been studied in detail. Nevertheless, the submicroscopic approach described in the present paper allows us to shed new light upon those phenomena. Indeed, we may apply the statistical mechanical approach developed in works (Krasnoholovets \& Lev, 2003; Krasnoholovets, 2001b; Krasnoholovets, 2004) to account for collective effects of cooling gases. This approach makes it possible to investigate how the quantum mechanical field (a substructure of the matter waves, i.e. inertons, carriers of particles' field of inertia) is able to interfere the collective behavior of atoms.

When atoms are cooled to the temperature where the de Broglie thermal wavelength $\lambda_{\text {th }}=h /\left(3 m k_{\mathrm{B}} \Theta\right)^{1 / 2}$ is comparable to the mead distance $g$ between atoms, the atomic wave functions overlap and the atoms by Ketterle (2002) "become a 'quantum soup' of indistinguishable particles". In such a "soup" atoms are found in the coherent state. However, submicroscopic mechanics states that the overlapping of inerton clouds of entities takes place much early at a higher temperature and a larger distance between atoms, because the overlapping is determined by relationship (1). The phenomenon of the whole coherent state in which the motion of all the atoms is synchronized appears when the de Broglie wavelength $\lambda_{\text {th }}$ exactly becomes equal to the distance $g$ between atoms. In this case the $(\mathbf{n}-\mathbf{g})$ th atom emits its inerton cloud that then is fully absorbed by the $\mathbf{n}$ th atom; the $\mathbf{n t h}$ atom emits its own cloud of inertons, which then is fully absorbed by the $(\mathbf{n}+\mathbf{g})$ th atom, etc. In other words, the coherent exchange of inerton clouds by the atoms when an inerton cloud emitted by one atom hops to the neighbor atom and is absorbed by it, we relate with the phenomenon of Bose-Einstein condensation.

Let us investigate whether the occurrence of clusters can be possible in a Bose-Einstein condensate. The action $S$ for the ensemble of $N_{\text {total }}$ interacting boson particles, which tend to clusterization with $N$ particles in a cluster, has the form (Krasnoholovets \& Lev, 2003)

$$
S \cong K \cdot\left\{\frac{1}{2}(a-b) N^{2}-\ln (N+1)+N \ln \xi\right\}
$$

where the functions $a$ and $b$ are determined in expressions (19) and (20) respectively. For simplicity, the repulsion potential can be taken in the form (17). The attraction potential should include at least three terms: 1) the dispersion potential of interatomic interaction, which is usually written as $-C_{6} / r^{6} ; 2$ ) a potential formed by a trap, which can be modeled by a harmonic potential and 3) the harmonic potential caused by small spatial oscillations of atoms near their equilibrium positions, i.e. inerton elastic interaction. So, the attraction and the repulsion potentials are

$$
\begin{gathered}
V_{\text {att }}(\rho)=C_{6} /(r \rho)^{6}-\frac{1}{2} \gamma_{\text {trap }} r^{2} \rho^{2}-\frac{1}{2} m \omega^{2}(\delta r)^{2} \rho^{2}, \\
V_{\text {rep }}(\rho)=V_{0 \text { rep }} / \rho^{12}
\end{gathered}
$$

where $m$ is the mass of an atom, $r$ is the distance between atoms, $\gamma$ is the effective force constant of the trap, $\omega$ is the cyclic frequency of proper oscillations of an atom, $\delta r$ is the appropriate amplitude and $\rho$ is the dimensionless distance parameter.

Substituting potentials (28) and (29) into functions (19) and (20) respectively, we then construct the action (18), 
which in the explicit form becomes

$$
\begin{gathered}
S=K \cdot\left\{\frac{1}{6} \frac{V_{0 \text { rep }}}{k_{\mathrm{B}} \Theta} N^{2}-\frac{3}{6} \frac{C_{6}}{r^{6} k_{\mathrm{B}} \Theta} N^{2}+\frac{3}{20} \frac{1}{k_{\mathrm{B}} \Theta}\left(\gamma_{\text {trap }} r^{2}+m \omega^{2} \delta r^{2}\right) N^{11 / 3}\right. \\
-\ln (N-1)+N \ln \xi\}
\end{gathered}
$$

Proper oscillations of atoms, which are characterized by the frequency $\omega$, are produced by the movement of the atoms. In other words, the origin of the frequency $\omega$ is produced by collisions of atoms with their inerton clouds (Krasnoholovets, 2002a): $\omega=2 \pi / 2 T$ where $T$ is the period of time between collisions of an atom and its inerton cloud (though in the present case we have to talk about collisions of the inerton cloud of an atom with the neighbor atom, Krasnoholovets \& Tane, 2006); the value of $T$ is related with the amplitude $\delta r$ of oscillations of an atom and its velocity $v, 1 / T=v / \delta r$ (because the amplitude $\delta r$ of oscillations of the atom is related to the atom's de Broglie wavelength $\lambda$ ). Since in the case of Bose-Einstein condensation the chemical potential of atoms we can put $\mu=0$, the fugacity $\xi=1$ and hence the last term in expression (30) reduces to zero.

The equation for the number of atoms combined in a cluster comes from the equation $\partial S / \partial N=0$, or explicitly

$$
N \cong\left(\frac{10}{33} \frac{9 C_{6} / r^{6}-4 V_{0 \text { rep }}}{\gamma_{\text {trap }} r^{2}+m \omega^{2} \delta r^{2}}\right)^{3 / 5} .
$$

Here in expression (31) in the first approximation the numerator, i.e. the difference between the attraction and repulsion energies at an equilibrium distance $r$ of interacting atoms, can be put $9 C_{6} / r^{6}-4 V_{0}$ rep $\approx 10 k_{\mathrm{B}} \Theta_{300} \cong 4.25 \times 10^{-20} \mathrm{~J}$, where $\Theta_{300}=300 \mathrm{~K}$, and we may neglect the trapping potential $\gamma_{\text {trap }} r^{2} / 2$ (nevertheless see Fig. 5). Let us assign numerical values to the parameters $\delta r$ and $\omega$ for the case of cesium atoms whose mass $m_{\mathrm{Cs}}=2.207 \times 10^{-25} \mathrm{~kg}$. Since the amplitude $\delta r$ of oscillations of an atom is associated with its de Broglie wavelength $\lambda$, we may write $\delta r=\lambda=h /\left(m_{\mathrm{Cs}} v\right)$.

However, in the case of dilute gases of atoms a lattice of atoms is not formed, as the overlapping of their inerton clouds is not strong enough. That is why the oscillation of atoms is caused only by their thermal motion: $v \approx v_{\text {th }}=\left(3 k_{\mathrm{B}} \Theta / m_{\mathrm{Cs}}\right)^{1 / 2} \cong 3 \times 10^{-3} \mathrm{~m} / \mathrm{s}$ where we put a typical temperature of Bose-Einstein condensate $\Theta=50 \mathrm{nK}$. So, $\delta r=\lambda_{\text {th }}=h /\left(m_{\mathrm{Cs}} v_{\mathrm{th}}\right) \cong 10^{-6} \mathrm{~m}$ and then the cycle frequency of atom oscillations becomes $\omega=\pi v / \delta r \cong 9.43 \times 10^{3} \mathrm{~s}^{-1}$. The abovementioned numerical values of the parameters allow the evaluation of the number of atoms that assemble in a Bose-Einstein cluster: $N \approx 1.95 \times 10^{5}$.

A variation of the parameters in expression (31) allows us to construct the dependence of the number of atoms in a cluster $N$ versus the de Broglie thermal wavelength, or amplitude $\delta r$, Fig. 5. In fact, these estimates are in line with experimental observations that fix up to around $10^{10}$ atoms being in the state of Bose-Einstein condensation (Phillips, 1998; Cornell \& Wieman, 2002).

Thus, the presented consideration makes it possible to account for the quantity of matter that is found in the condensed state: inter-atomic interactions subdivide the system of atoms to clusters but the cluster state is realized when the absolute value of an attraction potential starts to exceeds the thermal energy, $V_{\text {att }} \geq k_{\mathrm{B}} \Theta$. This inequality holds for the case calculated above: the attraction energy $\frac{1}{2} m_{\mathrm{Cs}} \omega^{2} \delta r^{2} \approx 1.7 \times 10^{-30} \mathrm{~J}$ exceeds the thermal energy $k_{\mathrm{B}} \Theta \approx 7 \times 10^{-31} \mathrm{~J}$.

When the drift/diffusive rate of ultracold atoms increases (a typical observed speed is around $1 \mathrm{~cm} / \mathrm{s}$ ), the thermal de Broglie wavelength $\lambda_{\text {th }}$ drops down, which must bring to rearrangement of atoms, because at such conditions the density of gas going up bringing Bose-Einstein clusters to collapse.

It seems it will be interesting to investigate the behavior of Bose-Einstein clusters affected by the inerton field, because, as it has been shown in the previous subsection, the absorption of outside mass is able to 'freeze' particles in the system studied and hence can stabilize the system. However, in the present case the frequency of oscillations $\omega$ is inversely proportional to the particle amplitude $\omega=\pi v_{\text {th }} / \delta r$ (the same as is the case of a free particle $\omega=\pi v / \lambda$ ). Because of that in the solution (31) the potential energy $m \omega^{2} \delta r^{2} / 2$ is reduced to $3 \pi^{2} k_{\mathrm{B}} \Theta$. So, no dependency on the amplitude $\delta r$. 
This means that atoms in Bose-Einstein condensates cannot be further frozen by exposure with an inerton field. Moreover, since the atoms do not practically interact, they will not absorb inertons. Incident inertons can be captured by a system of particles only in the case of a strong or at least intermediate particle-particle interaction; in this case absorbed inertons are aligned so that particles' overlapping inerton clouds tend to hold them together. In a lattice of particles the generation of inerton excitations follows the scheme posed in section 2 (see expression (13)). So in the potential energy $m \omega^{2} \delta r^{2} / 2$ the amplitude $\delta r$ is not cancelled but plays an important role of a receptor of inertons.

\section{Concluding remarks}

In the proposed work it has been argued that in addition to the vibratory motion of atoms in a substance a variation in their mass also occurs. Starting from first sub-atomic principles of the constitution of real space the mechanism of variation in mass of atoms/molecules in condensed matter is analyzed. It is shown that the wave function $\psi$ introduced in quantum mechanics by Schrödinger represents the field of inertia of the appropriate moving/vibrating entity (an atom or a molecule) in the substance in question. This field is characterized by carriers, quasi-particles (excitations) of the real space, called inertons and these excitations of space are responsible for the mass exchange between the substance's entities. The overlapping of real inerton clouds, i.e. corresponding wave functions determined in a phase space, results in the emission and re-absorption of inertons by vibrating atoms/molecules.

Emission and re-absorption of inertons by entities means that the mass of atoms in any substance is not a stationary parameter, but dynamic. The value of mass varies with anamplitude $\Delta m$ that is small in comparison with the rest mass of the atom. However, as has been shown in previous works (Krasnoholovets, Skliarenko \& Strokach, 2006; Andreev, Dovbeshko \& Krasnoholovets, 2007; Krasnoholovets, Kukhtarev \& Kukhtareva, 2006), the inerton field can be excited in some substances and is able to affect other substances inducing novel effects: change in mass rearranges entities, which tends to a peculiar secondary phase transition in the substance in question, namely, clusters. Moreover, the generated inerton field can be intensive, such that irradiated samples begin to change their chemistry, which has in fact been observed in the study of mixture of oil and methanol (Dekhtiaruk, Krasnoholovets \& Heighway, 2007).

The coherent emission and absorption of inerton clouds by nearest atoms supply deeper information on Bose-Einstein condensation of cool atoms. Instability of Bose-Einstein condensates with respect to expansion of atoms, a Feshbach resonance when a meta-stable composite sub system is temporary created, and other effects might be described in terms of the submicroscopic consideration as well, not only in the framework of the Gross-Pitaevski (Gross, 1961,1963; Pitaevski, 1961) quantum mechanical equation. The point is that a Bose-Einstein condensate cluster can be treated not only as a whole continuous object, which is described by a unified wavefunction $\psi$, but also as a dynamic system of many coherently oscillating entities, like a nucleus that consists of many nucleons. Such approach would bring some new results in the description of Bose-Einstein condensates, the more so that it is completely deterministic owing to carriers, i.e. inertons, which establish a short-range interaction between entities.

Thus effects induced by inerton fields in condensed media are quite important and, therefore, further studies of these fields and their interaction with substances promise the discovery of new physical phenomena and open a gateway to new advanced technologies.

\section{Acknowledgements}

The author thanks greatly Dr. I. Gandzha for the assistance in numerical calculations.

\section{References}

Andreev, E., Dovbeshko, G. \& Krasnoholovets, V. (2007). The study of influence of the Teslar technology on aqueous solution of some biomolecules. Research Letters in Physical Chemistry, Article ID 94286, p5. [Online] Available: http://www.hindawi.com/getarticle.aspx?doi=10.1155/2007/94286.

Agranovich, V. M., \& Galanin, M. D. (1978). Transfer of energy of electronic excitation in condensed media, Nauka, Moscow, p. 27; in Russian.

Bounias, M., \& Krasnoholovets, V. (2003a). Scanning the structure of ill-known spaces: Part 1. Founding principles about mathematical constitution of space, Kybernetes: The International Journal of Systems and Cybernetics, 32, Nos. 7/8, 945-975; arXiv.org: physics/0211096.

Bounias, M., \& Krasnoholovets, V. (2003b). Scanning the structure of ill-known spaces: Part 2. Principles of construction of physical space, ibid., 32, Nos. 7/8, 976-1004; arXiv: physics/0212004. 
Bounias, M., \& Krasnoholovets, V. (2003c). Scanning the structure of ill-known spaces: Part 3. Distribution of topological structures at elementary and cosmic scales, ibid., 32, Nos. 7/8, 1005-1020; arXiv: physics/0301049.

Bounias, M., \& Krasnoholovets, V. (2004). The universe from nothing: A mathematical lattice of empty sets. International Journal of Anticipatory Computing Systems, 16, 3-24; arXiv.org: physics/0309102

Cohen-Tannoudji, C. (1998). Manipulating atoms with photons, 1997 Nobel Lecture in Physics. Reviews of Modern Physics, 70, No. 3, 707-719.

Cornell, E. A., \& Wieman, C. E. (2001). Bose-Einstein condensation in a dilute gas, the first 70 years and some recent experiments, 2001 Nobel Lecture in Physics. Reviews of Modern Physics, 74, No. 3, 875-893.

Chu, S. (1998). The manipulation of neutral particles, 1997 Nobel Lecture in Physics. Review in Modern Physics, 70, No. 3, 685-706.

De Broglie, L. (1982). Les incertitudes d'Heisenberg et l'interprétation probabiliste de la méchanique ondulatoire, Paris: Gauthier-Villars, Bordas, (Chapter 2, sect. 4.) (Russian translation: Mir, Moscow, 1986; pp. $50-52)$.

Dehmelt, H., \& Ekstrom, P. (1973). Proposed g-2/dvz experiment on stored single electron or positron. Bulleten of American Physical Society, 18, 727.

Dekhtiaruk, V., Krasnoholovets, V., \& Heighway, J. (2007). Biodiesel manufacture, International patent No. PCT/GB2007/001957 (Intern. filing date 25 May 2007; London, UK).

Didkovsky, V., \& Krasnoholovets, V., A first step of inerton astronomy, to be submitted.

Emoto, M. (2005). The secret life of water, Beyond Word Publishing Inc. \& Simon and Schuster Inc. (Russian translation: Minsk: Popurri Ltd., 2006).

Feder, J. (1988). Fractals, New York and London: Plenum Press, (Chapter 2).

Gross, E. P. (1961). Structure of quantized vortex, Il Nuovo Cimento, 20, 454-477.

Gross, E. P. (1963). Hydrodynamics of a superfluid condensate. Journal of Mathematical Physics, 4, No. 2, 195-207.

Ketterle, W. (2002). When atoms behave as waves: Bose-Einstein condensation and the atom laser, 2001 Nobel Lecture in Physics. Reviews of Modern Physics, 74, No. 4, 1131-1151.

Kittel, C. (1978). Introduction to solid state physics, Moscow: Nauka; Russian translation.

Krasnoholovets, V. (2001a). On the theory of the anomalous photoelectric effect stemming from a substructure of matter waves. Indian Journal of Theoretical Physics, 49, No. 1, 1-32; arXiv: quant-ph/9906091.

Krasnoholovets, V. (2001b). Collective dynamics of hydrogen atoms in the $\mathrm{KIO}_{3} \cdot \mathrm{HIO}_{3}$ crystal dictated by a substructure of the hydrogen atoms' matter waves, arXiv: cond-mat/0108417.

Krasnoholovets, V. (2002a). Submicroscopic deterministic quantum mechanics, International Journal of Computing Anticipatory Systems, 11, 164-179; arXiv: quant-ph/0109012.

Krasnoholovets, V. (2002b). Gravitation as deduced from submicroscopic quantum mechanics, arXiv: hep-th/0205196.

Krasnoholovets, V. (2003). On the nature of the electric charge, Hadronic Journal Supplement, 18, No. 4, 425-456; arXiv: physics/0501132.

Krasnoholovets, V. (2004). Clusterization of water molecules as deduced from statistical mechanical approach. Central European Journal of Physics, 2, No. 4, 698-708.

Krasnoholovets, V. (2008). Reasons for the gravitational mass and the problem of quantum gravity, to be published in M. Duffy, J. Levy \& V. Krasnoholovets (Eds.). Ether, spacetime and cosmology, Liverpool: PD Publications, pp. 407-438.

Krasnoholovets, V., \& Byckov, V. (2000). Real inertons against hypothetical gravitons. Experimental proof of the existence of inertons. Indian Journal of Theoretical Physics, 48, No. 1, 1-23; arXiv: quant-ph/0007027.

Krasnoholovets, V., \& Lev, B. (2003). Systems of particles with interaction and the cluster formation in condensed matter. Condensed Matter Physics, 6, No. 1, 67-83; arXiv: cond-mat/0210131. 
Krasnoholovets, V., Kukhtarev, N., \& Kukhtareva, T. (2006). Heavy electrons: Electron droplets generated by photogalvanic and pyroelectric effects. International Journal of Modern Physics B, 20, No. 16, 2323-2337; arXiv.org: 0911.2361[quant-ph].

Krasnoholovets, V., Skliarenko, S., \& Strokach, O. (2006a). On the behavior of physical parameters of aqueous solutions affected by the inerton field of Teslar ${ }^{\circledR}$ technology. International Journal of Modern Physics B, 20, No. 1, 1-14; arXiv.org:0810.2005[physics.gen-ph].

Krasnoholovets, V., Skliarenko, S., \& Strokach, O. (2006b). The study of the influence of a scalar physical field on aqueous solutions in a critical range. Journal of Molecular Liquids, 127, 50-52.

Krasnoholovets, V., \& Tane, J.-L. (2006). An extended interpretation of the thermodynamic theory including an additional energy associated with a decrease in mass. International Journal of Simulation and Process Modelling, 2, Nos. 1/2, 67-79; arXiv: physics/0605094.

Phillips, W. D. (1998). Laser cooling and trapping of neutral atoms, 1997 Nobel Lecture in Physics. Reviews of Modern Physics, 70, No. 3, 707-719.

Pitaevski, L. P. (1961). Vortex lines in an imperfect Bose gas, Zhurnal Eksperimentalnoy i Teoreticheskoy Fiziki, 40, 646-649 [English translation: Soviet Physics JETP, 13, 451-454 (1961)].

Santilli, R. M. (2001). Foundations of hadronic chemistry with applications to new clean energies and fuels, Boston-Dorderecht-London: Kluwer Academic Publishers, (Chapter 8).

Strobbe, H., \& Peschel, G. (1997). Experimental determination of the static permittivity of extremely thin liquid layers of water dependent on their thickness. Colloid and Polymer Science, 275, 162-69.

Van Dyck, Jr., R. S., Ekstrom, P., \& Dehmelt, H. (1976). Axial, magnetron, cyclotron and spin-cyclotron beat frequencies measured on single electron almost at rest in free space (Geonium). Nature, 262, 776-777.

Van Dyck, Jr., R. S., Schwinberg, O. B., \& Dehmelt, H. G. (1986). Electron magnetic moment from geonium spectra: Early experiments and background concepts. Physical Review D, 34, 722-736.

Wineland, D., Ekstrom, P. \& Dehmelt, H. (1973). Monoelectron oscillator. Physical Review Letters, 31, 1279-1282.

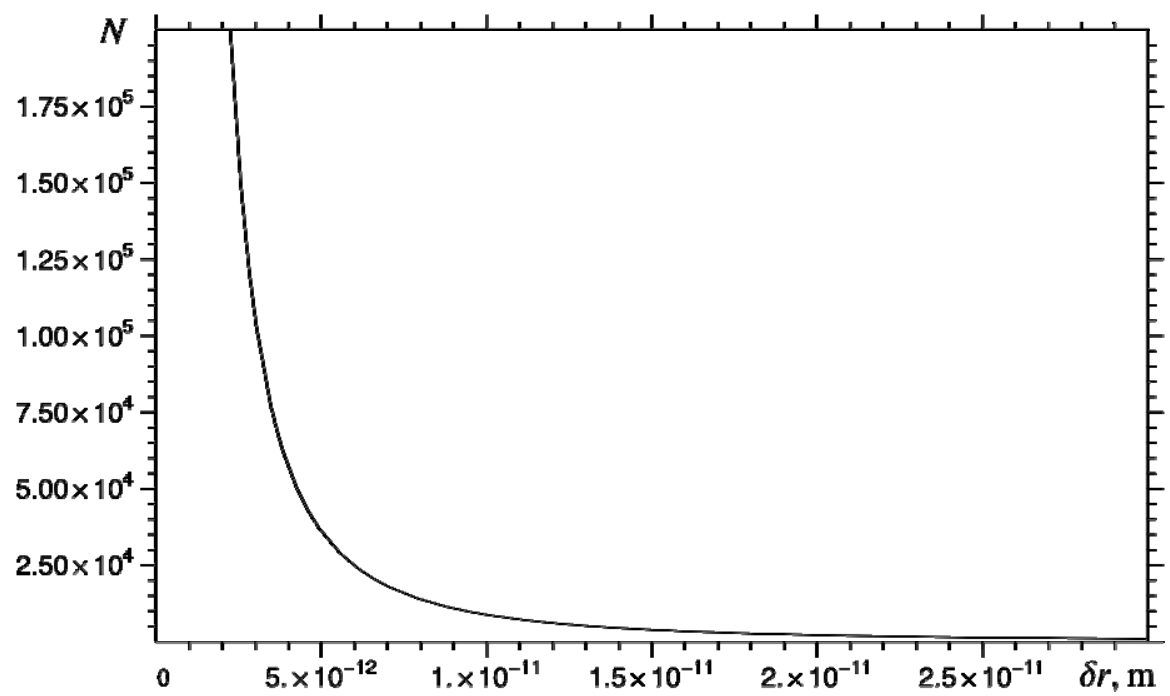

Figure 1. Number of molecules $N$ in a cluster versus amplitude $\delta r$ of molecule oscillations, i.e. the solution of Eq. (22) as the function of amplitude $\delta r$. 


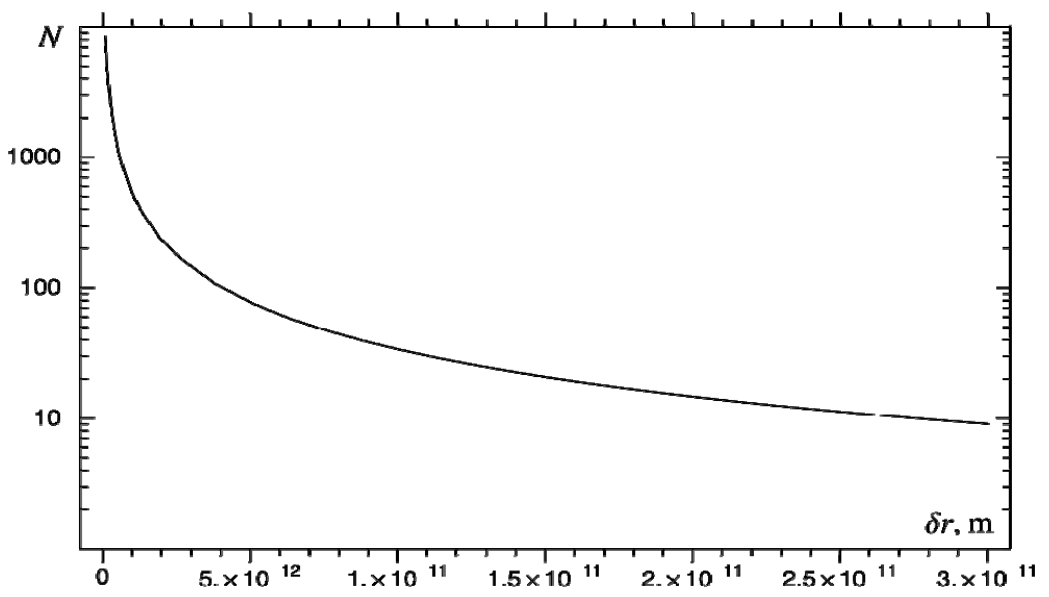

Figure 2. Numerical solution $N$ versus $\delta r$ (25); values of the parameters are the same as above: $V_{0}=4.25 \times 10^{-20} \mathrm{~J}$ and $\gamma=1 \mathrm{~N} / \mathrm{m}$.
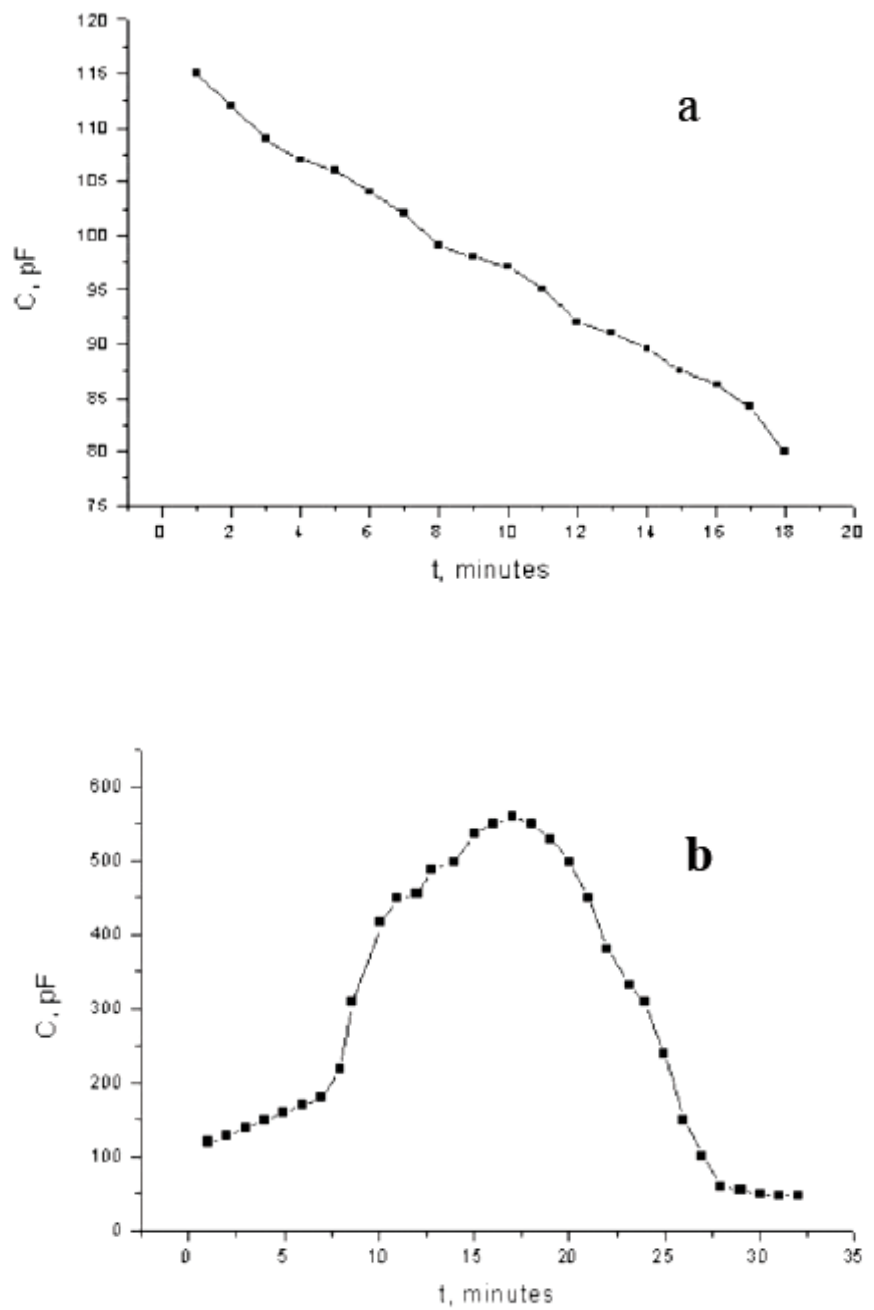

Figure 3. Capacity of the 50\%-aqueous solution of alcohol found in an open cuvette as a function of time: (a) without an outside influence; (b) under the influence of the inerton field generated by a signal generator of the Teslar $^{\circledR}$ technology; from (Krasnoholovets, Skliarenko \& Strokach, 2006). 


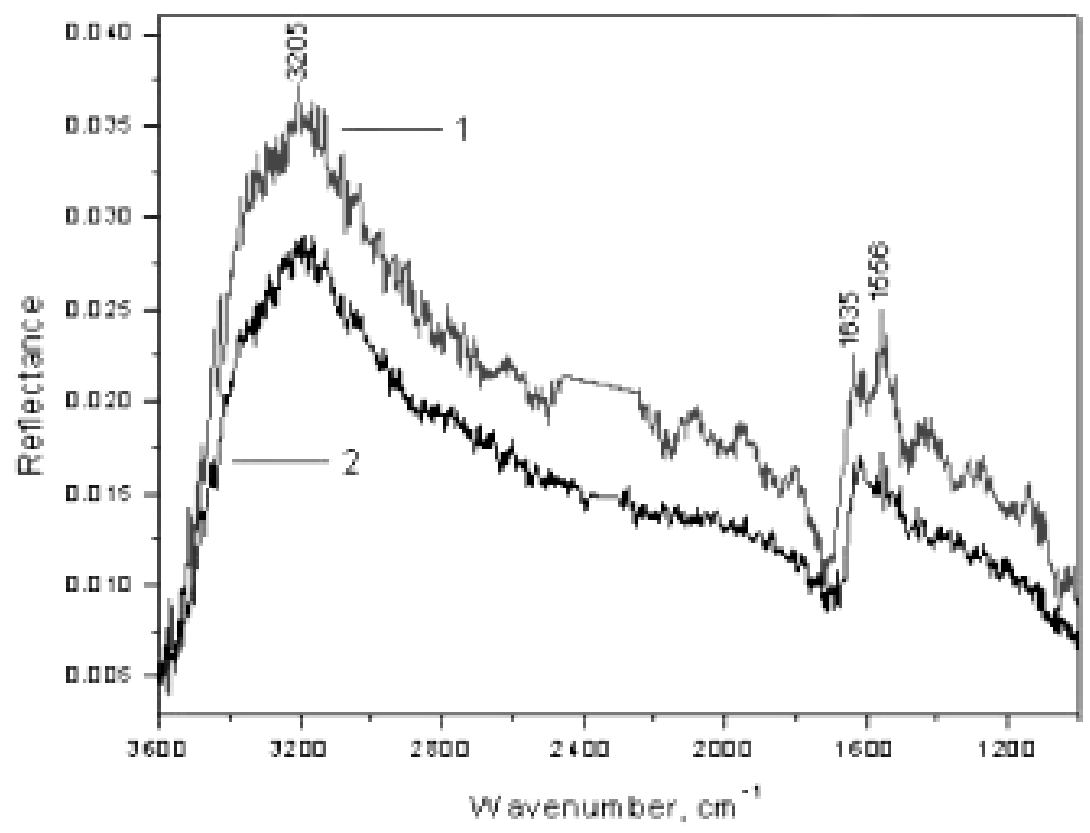

Figure 4. IR-RAS spectra of $\mathrm{H}_{2} \mathrm{O}_{2}$ solution just after pouring it in the cuvette. 1 (upper curve): the sample did not undergo an outside influence; 2 (lower curve): the sample was affected by a signal generator of the Teslar ${ }^{\circledR}$ technology; from (Krasnoholovets \& Tane, 2006).

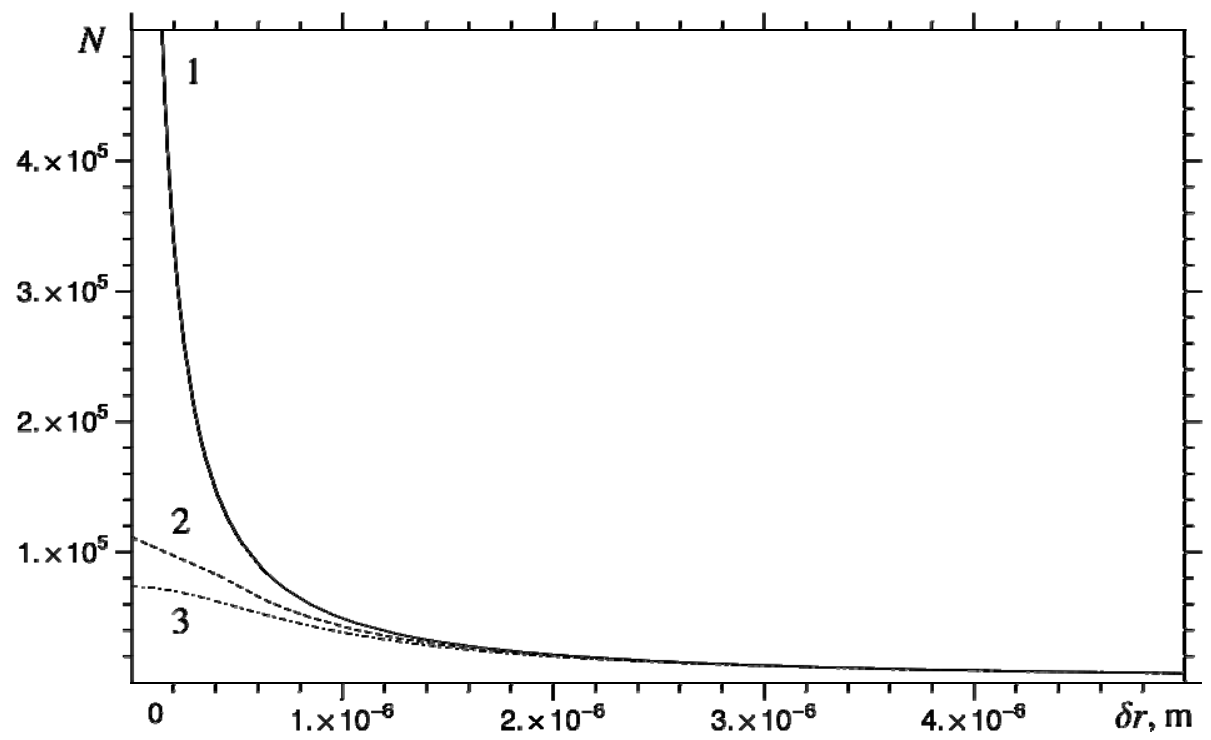

Figure 5. Number of atoms $N$ in a Bose-Einstein condensate cluster versus amplitude $\delta r$ of thermal motion of atoms, i.e. the solution of Eq. (31) as the function of the de Broglie thermal wavelength $\delta r=\lambda$ trap $=h /\left(m_{\text {th }} v_{\text {th }}\right)=h /\left(3 k_{\mathrm{B}} \Theta m_{\mathrm{Cs}}\right)^{1 / 2}$. Here, the double harmonic trapping potential $\gamma_{\text {trap }} r^{2}=0$ (curve 1$), 5 \times 10^{-29}$ $\mathrm{J}$ (curve 2) and $5 \times 10^{-28} \mathrm{~J}$ (curve 3 ). 